\title{
A Study on Association between Intraocular Pressure and Myopia
}

\author{
Pallavi Bangalore Acharlu¹, Prashul Rayi M. K.'2, Vijay Kumar Srivastava ${ }^{3}$ \\ ${ }^{1}$ Department of Ophthalmology, MVJ Medical College and Research Hospital, Hoskote, Karnataka, India. \\ ${ }^{2}$ Department of Ophthalmology, MVJ Medical College and Research Hospital, Hoskote, Karnataka, India. \\ ${ }^{3}$ Department of Ophthalmology, MVJ Medical College and Research Hospital, Hoskote, Karnataka, India.
}

\section{ABSTRACT}

\section{BACKGROUND}

High intraocular pressure is a major risk factor for Glaucoma in Myopic patients. The aim of this study is to study the association between IOP and myopia.

\section{METHODS}

A hospital-based study was done from December 2018 to May 2019 in 150 eyes. Patients in the age group of 10 - 50 yrs. were studied. Patients were divided into four groups according to their refractive status. Group 0- emmetropia (+0.5 D to -0.5 D), Group 1- low myopia ( -0.75 to -3.00 D), Group 2- moderate myopia ( -3.25 D to$5.00 \mathrm{D})$ and Group 3- high myopia (>-5.00 D). Examinations like slit lamp, retinoscopy, gonioscopy, fundus examination were done. IOP was measured using Perkins tonometer.

\section{RESULTS}

Group 0 included 64 eyes, Group 1 included 44 eyes, Group 2 had 26 eyes, and Group 3 had 16 eyes. There is no significant difference in IOP between Group 0 and Group 1 but IOP was found to be higher in Group 2 and 3.

\section{CONCLUSIONS}

There is significant correlation between intra ocular pressure and high/moderate myopia than with emmetropes and low-grade myopia, thus increasing the chances of glaucoma in patients with high/moderate myopia.

\section{KEY WORDS}

Myopia, Intraocular pressure, Glaucoma, Refractive Error

\section{Corresponding Author:} Prashul Rayi M. K., No. 34/8, 1st Floor, Syndicate Bank Quarters, Nandini layout, Bangalore-560096, Karnataka, India.

E-mail: prashulrayi@gmail.com

DOI: $10.14260 /$ jemds/2020/327

Financial or Other Competing Interests: None.

How to Cite This Article: Acharlu PB, Rayi PMK, Srivastava VK. A study on association between intraocular pressure and myopia. J. Evolution Med. Dent. Sci. 2020;9(18):1500-1503, DOI: $10.14260 /$ jemds/2020/327

Submission 03-03-2020, Peer Review 17-04-2020, Acceptance 23-04-2020, Published 04-05-2020. 


\section{BACKGROUND}

Myopia or near-sightedness is a refractive error in which rays of incident light from infinity are focused in front of retina with accommodation being at rest. ${ }^{1}$ it is a condition in which close objects are seen clearly, but objects farther away appear blurred. Myopia occurs if the eyeball is too long or the cornea, the clear front cover of the eye, has too much curvature. As a result, the light entering the eye is not focused correctly and distant objects look blurred. A common sign of myopia is difficulty with the clarity of distant objects like a movie or TV screen or the chalkboard in school going children. The severe myopia (high degree myopia) has more likely to develop eye disorders in later life. These might include: 1) Retinal detachment- the retina begins to pull away from the blood vessels that supply it with oxygen and nutrients; if left untreated, retinal detachment can cause permanent vision loss; 2) Glaucoma-pressure builds up inside the eye which, if left untreated, can also pose a threat to vision; 3) Cataractsopacities present in the lens; 4) Macular degeneration-the central section of the retina (the macula) becomes damaged, leading to some loss of central vision. ${ }^{2}$ All these are causes for avoidable blindness. Myopia is one of the most common treatable ocular condition. In most cases, normal vision could be restored with eyeglasses or contact lenses and refractive surgeries.

Intra ocular pressure (IOP) is the fluid pressure inside the eye. Intraocular pressure plays an important role in the pathogenesis of glaucoma and has been hypothesized to be one of the several factors implicated in the pathogenesis of glaucoma.3,4,5 Few studies has shown that myopia is important risk factor for POAG.6,7 Most population based study in adults have found significant relation between IOP and myopia. ${ }^{8,9}$ Although some dispute still exist in few clinical based studies. ${ }^{10,11}$

Therefore, we aim to investigate the association of IOP and myopia in a population presenting to Ophthalmology OPD at Hoskote.

\section{METHODS}

This is a hospital based prospective observational study done in the department of Ophthalmology, MVJ Medical College and Research Hospital over a period of 6 months from December 2018 to May 2019. In our OPD, 150 eyes of 120 patients were included in the study after taking their informed consent. A total of 120 patients (150 eyes) of the mean age 10 to 50 yrs. were taken. Patients were divided into four groups according to their refractive status. Group 0 Emmetropia (+0.5 D to $-0.5 \mathrm{D})$, Group 1 low myopia ( -0.75 to $-3.00 \mathrm{D})$, Group 2 moderate myopia ( -3.25 D to-5.00 D) and Group 3 high myopia (>-5.00D). Interviews to obtain details of Diabetes, Hypertension, and information about eye disease was done. In order to ascertain inclusion of healthy eyes only, patients with Astigmatism and Hyperopia >+0.5 DS, on ocular medication such as antiglaucoma medications, steroids, family history of Glaucoma, presence of Pseudoexfoliation, history of previous ocular surgeries, ocular trauma were excluded from the study. Myopia associated with other systemic diseases and ocular diseases were excluded from the study.

A detailed ocular examination was made using slit lamp. Best corrected visual acuity was measured using Snellen's visual acuity chart. Retinoscopy and Refraction was done. Gonioscopic examination was done with Goldman three mirror Gonioscope. Detailed fundus evaluation was done after full dilatation of pupil by instilling tropicamide plus eye drops at an interval of 5 minutes for three times and viewed after 45 minutes using Direct and Indirect Ophthalmoscope. The Examiner inspected for the Optic disc size, shape, colour, vascularity and cup disc ratio. Intraocular pressure was measured using a handheld Perkins tonometer in the OPD after instillation of a drop of $0.5 \%$ paracaine in the eyes of the patient and after staining with $2 \%$ fluorescein. Measurements adhered to protocols; the patient was seated, with eyes in the primary position, eyelids or eyelashes did not obscure the applanation circle and the alignment spot was centred and focused in the alignment circle before applanation. IOP was assessed for individual eyes. One reading was taken. All the patients were examined by the same Ophthalmologist. The demographic Details of the patient along with Ophthalmological findings were entered in the Excel sheet.

Selected patients were divided into four groups according to their refractive status. The four categories were:- Group 0 Emmetropia (+0.5 DS to -0.50 DS), Group 1 low myopia ( -0.75 DS to -3.00 DS), Group 2 moderate myopia ( -3.25 DS to-5.00 DS), Group 3 high myopia (>-5.00 DS).

\section{Data Analysis}

Data collected was entered into MS Excel and tables/ charts are generated using MS word. Qualitative variables are presented as percentages and continuous variables are presented as Mean \pm SD and independent t-test is used to compare IOP and myopia between control and other groups. $P$ value $<0.05$ was considered as statistically significant.

\section{RESULTS}

A total of 120 patients (150 eyes) were included in the study in the age group of 10 to 50 years. Group 0 has 64 eyes making 43\%, Group 1 has 44 eyes with 29\%, Group 2 has 26 eyes with 17\%, and Group 3 has 16 eyes with 11\% (table 1).

\begin{tabular}{|ccc|}
\hline Study Population & Distribution & Percentage (\%) \\
Group 0 (+0.5 D - - 0.5 D) & 64 & 43 \\
Group 1 ( -0.75 D TO - 3.00 D $)$ & 44 & 29 \\
Group 2 ( -3.25 T0 -5.00 D) & 26 & 17 \\
Group 3 (>-5.00 D) & 16 & 11 \\
\hline \multicolumn{2}{|c|}{ Table 1. Distribution of the Refractive Error } \\
among the Different Study Groups \\
\hline
\end{tabular}

Group 0 was taken as the control group. The mean IOP of Group 0 was 12.66 mm Hg (SD-2.10), Group 1 was $12.27 \mathrm{~mm}$ $\mathrm{Hg}$ (SD- 2.22), Group 2 was $15.07 \mathrm{~mm} \mathrm{Hg}$ (SD - 3.21), Group 3 was $18.30 \mathrm{mmHg}$ (SD- 3.24) (table 2). 


\begin{tabular}{|ccc|}
\hline Study Group & Mean IOP (mm Hg) & Standard Deviation \\
Group 0 & 12.6562 & 2.1098 \\
Group 1 & 12.2727 & 2.2242 \\
Group 2 & 15.0769 & 3.2115 \\
Group 3 & 18.3000 & 3.2429 \\
\hline Table 2. Mean IOP among the Different Study Groups \\
\hline
\end{tabular}

There is no significant difference in IOP between Group 0 and Group 1 but IOP was found to be higher in Group 2 and 3.Thus Group 2 and 3 are found to be statistically significant as $p$ value is $<0.05$ (table 3 ).

\begin{tabular}{|cccc|}
\hline Control Group & Study Group & Mean Difference in IOP & Significance \\
\multirow{2}{*}{ Group 0 } & Group 1 & 0.3835 & 0.3710 \\
& Group 2 & -2.4207 & 0.0011 \\
& Group 3 & -5.6438 & 0.001 \\
\hline \multicolumn{3}{|c|}{ Table 3. Comparison of IOP in the Study Groups } \\
\hline
\end{tabular}

\section{DISCUSSION}

The results in this study found a positive correlation between intraocular pressure and myopic patients in Group 2 and 3. The intraocular pressure was significantly higher in high myopic groups and moderate myopic groups compared to emmetropia and low myopic groups. Some studies noted no difference in IOP in refractive error status. ${ }^{12}$ various methods and samples may be the reason for difference in results in different studies. One of the hypothesis for increased relation of myopia with increasing IOP is the increased stress of global wall and decreased ocular rigidity in the Myopic eyes. ${ }^{13}$ Increased Intraocular pressure is related to an increased stress of global wall and decreased ocular rigidity in Myopic eyes. ${ }^{13}$ Friedman has proposed a mathematical model that suggests the Myopic eye is under greater stress than emmetropic eye with the same IOP. ${ }^{14}$ The incidence of increased chance of Open Angle Glaucoma in myopia may be due to stretching and tractional vectors which are not evenly distributed across the Myopic disc, or due to the reduced RNFL (Retinal Nerve Fiber Layer) thickness in myopia. ${ }^{14}$

Nesterov A et al postulated that, in myopic eyes the ciliary body is in relatively posterior position in relation to canal of Schlemm so that it has less mechanical advantage in widening the spaces in the trabecular meshwork during accommodation. Both glaucoma and myopia have a strong familial basis and may share a common genetic link. An early report indicated that people with high myopia are more likely to be steroid responders than those in genetic community. ${ }^{15}$ Recently, the gene coding for a trabecular meshworkinduced-glucocorticoid response protein in the GLC1A locus on chromosome 1q21-q31 was identified and found in 3.9\% of glaucoma population compared to $0.3 \%$ of general population. ${ }^{16}$ It is possible that this and other Glaucoma genes may be represented more frequently in persons with myopia.

Assessment of IOP alone can prove ambiguous for identifying glaucoma in high myopic patients due to the fact that IOP is significantly dependent on corneal thickness. Corneal thickness varies according to ethnic group, and is characteristically thin in myopic eyes, making them more likely to test negative for IOP elevation. ${ }^{17}$ The main limitation of this study would be that the axial length, corneal curvature and central corneal thickness were not measured. Therefore, the role of these factors on the refraction related mechanics of the globe were not known. The age and gender of these groups have not been matched which may have an influence on the results

Early detection of any pathology provides the greatest potential for prevention; this pertains equally to myopia and myopia-related Glaucoma. One of the most important approaches for monitoring early glaucoma changes in high Myopes is to perform routine visual field testing. At present, ophthalmologists may err on the side of performing visual field tests only when excessive optic nerve cupping or asymmetry in the optic nerve cupping between two eyes is apparent, but visual field testing should be used more routinely in high-risk myopic individuals to facilitate detection of early glaucomatous changes. The need for a systematic approach to estimate association between myopia and Glaucoma will can be addressed with consensus use of the Disc Damage Likelihood Scale, a new system for estimating the relationship between optic disc size, neuroretinal rim, and cup/disc ratio and assessing Glaucomatous damage of the optic disc in correlation with the degree of visual field loss. ${ }^{18}$

\section{CONCLUSIONS}

There is significant correlation between intra ocular pressure and high/moderate myopia than with emmetropes and lowgrade myopia, thus increasing the chances of glaucoma in patients with high/moderate myopia.

\section{REFERENCES}

[1] Chaudhari Z, Vanathi M. Postgraduate ophthalmology. 1st edn. Jaypee Brothers Medical Publishers (P) Ltd., 2012: p. 46-7.

[2] Yu L, Li ZK, Gao JR, et al. Epidemiology, genetics and treatments for myopia. Int J Ophthalmol 2011;4(6):65869.

[3] Sommer A, Tielsch JM, Katz J, et al. Relationship between intraocular pressure and primary open angle glaucoma among white and black Americans. The Baltimore Eye Survey. Arch Ophthalmol 1991;109(8):1090-5.

[4] Shiose Y, Kitazawa Y, Tsukahara S, et al. Epidemiology of glaucoma in Japan--a nationwide glaucoma survey. Jpn J Ophthalmol 1991;35(2):133-55.

[5] Bonomi L, Marchini G, Marraffa M, et al. Prevalence of glaucoma and intraocular pressure distribution in a defined population. The Egna-Neumarkt Study. Ophthalmology 1998;105(2):209-15.

[6] Marcus MW, De Vries MM, Montolio JFG, et al. Myopia as a risk factor for open-angle glaucoma: a systematic review and meta-analysis. Ophthalmology 2011;118(10):1989-94.e2. 
[7] Kuzin AA, Varma R, Reddy HS, et al. Ocular biometry and open-angle glaucoma: the Los Angeles Latino Eye Study. Ophthalmology 2010;117(9):1713-9.

[8] Quinn GE, Berlin JA, Young TL, et al. Association of intraocular pressure and myopia in children. Ophthalmology 1995;102(2):180-5.

[9] Abdalla MI, Hamdi M. Applanation ocular tension in myopia and emmetropia. $\mathrm{Br} \mathrm{J}$ Ophthalmol 1970;54(2):122-5.

[10] David R, Zangwill LM, Tessler Z, et al. The correlation between intraocular pressure and refractive status. Arch Ophthalmol 1985;103(12):1812-5.

[11] Daubs JG, Crick RP. Effect of refractive error on the risk of ocular hypertension and open angle glaucoma. Trans Ophthalmol Soc U K 1981;101(1):121-6.

[12] Lee AJ, Saw SM, Gazzard G, et al. Intraocular pressure associations with refractive error and axial length in children. Br J Ophthalmol 2004;88(1):5-7.
[13] Schmid KL, Li RWH, Edwards MH, et al. The expandability of the eye in childhood myopia. Curr Eye Res 2003;26(2):65-71.

[14] Friedman B. Stress upon the ocular coats: effects of scleral curvature scleral thickness and intra-ocular pressure. Eye Ear Nose Throat Mon 1966;45(9):59-66.

[15] Podos SM, Becker B, Morton WR. High myopia and primary open-angle glaucoma. Am J Ophthalmol 1966;62(6):1038-43.

[16] Stone EM, Fingert JH, Alward WL, et al. Identification of a gene that causes primary open angle glaucoma. Science 1997;275(5300):668-70.

[17] Bron AM, Creuzot-Garcher C, Goudeau-Boutillon S, et al. Falsely elevated intra Ocular pressure due to increased central corneal thickness. Graefes Arch Clin Exp Ophthalmol 1999;237(3):220-4.

[18] Dervisevic E, Pavljasevic S, Dervisevic A, et al. Challenges in early glaucoma detection. Med Arch 2016;70(3):2037. 